\title{
PERFIL SOCIOECONÔMICO E ANTROPOMÉTRICO DE CANDIDATAS À CIRURGIA BARIÁTRICA PELO SISTEMA ÚNICO DE SAÚDE
}

\section{Karina Quesada}

$\mathrm{MsC}$, Docente do curso de Medicina e Nutrição da Universidade de Marília (UNIMAR), Brasil.

\section{Cláudia Rucco Penteado Detregiachi}

PHD, Docente do curso de Medicina e Nutrição da Universidade de Marília (UNIMAR), Brasil.

\section{Sandra Maria Barbalho}

PHD, Docente do curso de Medicina da Universidade de Marília (UNIMAR)e Faculdade de Tecnologia em Alimentos de Marília, Brasil.

E-mail: smbarbalho@gmail.com

\section{Maria Rita Marques Oliveira}

PHD, Docente da Universidade Estadual Paulista (UNESP), Brasil.

\section{Irineu Rasera}

Médico Cirurgião do Centro de Gastroenterologia e Cirurgia da Obesidade, Clínica Bariátrica, Brasil.

\section{Eliege Carolina Vaz}

Nutricionista pela Universidade de Marília (UNIMAR), Brasil.

\section{Ricardo de Alvares Goulart}

Médico Cirurgião-Gastroenterologista da Associação Beneficente do Hospital Universitário da UNIMAR, Brasil.
RESUMO: A cirurgia bariátrica é considerada eficaz no controle e tratamento da obesidade grave, tendo como principais benefícios a melhora no perfil das comorbidades e consequente melhora na qualidade de vida. $\mathrm{O}$ objetivo deste estudo foi avaliar o perfil socioeconômico e antropométrico de obesas candidatas à cirurgia bariátrica. Foram estudadas cem pacientes do sexo feminino, com idade entre 20 e 45 anos, cadastradas pelo Sistema Único de Saúde. Entrevista individual foi realizada para a coleta dos dados. O índice de massa corporal foi calculado e classificado segundo a Sociedade Americana de Cirurgia Bariátrica. A classificação econômica foi estabelecida de acordo com a Associação Brasileira de Empresas de Pesquisa. Os resultados apresentaram média de idade de 33,3 $\pm 6,0$ anos; valores médios \pm DP de índice de massa corporal de $45,75 \pm 6,05 \mathrm{Kg} / \mathrm{m}^{2}$, prevalecendo o diagnóstico de obesidade mórbida (68\%). Segundo a classificação econômica, a maioria das mulheres pertence à classe C, o que corresponde a uma renda média familiar de $\mathrm{R} \$ 927,00$. Das entrevistadas, $45 \%$ são casadas e $66 \%$ têm escolaridade igual ou superior ao ensino médio completo, sendo que os trabalhos domésticos representam a atividade de maior frequência (28\%). Quanto ao início da obesidade, 38\% desenvolveram na infância. A hipertensão arterial foi a comorbidade de maior predomínio (48\%). Conclui-se que apesar da maioria das mulheres estar dentro dos padrões para indicação do procedimento cirúrgico, em uma parcela dela, uma busca precoce por essa alternativa ocorre sem que atinjam níveis mais exacerbados de IMC e, em alguns casos, sem a realização de tratamentos clínicos prévios com tratamentos conservadores.

PALAVRAS-CHAVE: Antropometria; Cirurgia Bariátrica; Obesidade.

\section{SOCIO-ECONOMIC AND ANTHROPOMETRIC PROFILE OF CANDIDATES FOR BARIATRIC SURGERY BY THE NATIONAL HEALTH SERVICE}

ABSTRACT: Bariatric surgery is efficient in the control and treatment of serious obesity. Its great assets are the lessening of co-morbidities and improvement in life quality. Current analysis evaluates the socioeconomical and anthropometric profile of obese candidates for bariatric surgery. One hundred female patients, aged between 20 and 45 years, were selected by the National Health Service and an individual interview was undertaken for data collection. Body mass index was calculated and classified according to the American Society of Bariatric Surgery. Economic classification was established according to the Brazilian Association of Research Firms. Results showed a mean age of $33.3 \pm 6.0$ years; mean rates $\pm S D$ of body mass index of $45.75 \pm 6.05 \mathrm{Kg} /$ $\mathrm{m}^{2}$, predominantly diagnosis for morbid obesity (68\%). According to economic classification, most females belong to class $\mathrm{C}$, corresponding 
to a mean family earning of $\mathrm{R} \$ 927.00$. Further, $45 \%$ are married and $66 \%$ have a high school diploma, with house chores as their main activity (28\%). Obesity started during childhood in $38 \%$ of the interviewed, with arterial hypertension as the predominant co-morbidity (48\%). Results showed that, although most females were within surgery standards, a certain percentage decided early for such an alternative without reaching higher BMI. In some cases, surgery was done without the previous conservative treatments.

KEY WORDS: Bariatric Surgery; Obesity; Anthropometry.

\section{INTRODUÇÃO}

Há consenso na literatura que a sociedade como um todo está passando por uma transição nutricional e que a obesidade tem se tornado um grave problema de saúde pública tanto em países desenvolvidos como em desenvolvimento. Estas transições incluem modificações na dieta e redução da atividade física que têm consequências negativas como o sobrepeso e a obesidade. Estas condições afetam aproximadamente $50 \%$ da população adulta e aproximadamente $30 \%$ da população infantil sendo considerada pela Organização Mundial da Saúde como uma epidemia global (RUIZ et al., 2015; VARELA-MOREIRAS et al., 2013; RIGBY, 2013; WHO, 2006). No Brasil há estimativa de 17,9\% de adultos obesos, sem diferença entre os sexos, além de 49,1\% das mulheres e $56,5 \%$ dos homens com sobrepeso (BRASIL, 2014).

O aumento do peso corporal é um fator de risco para hipertensão, hipercolesterolemia, diabetes mellitus, doenças cardiovasculares e algumas formas de câncer, contribuindo substancialmente para a morbidade e mortalidade da população. Com isso, o impacto da obesidade poderá ser cada vez mais alarmante visto que tem aumentado em todas as idades (MARQUES et al., 2015; LI; KUH; POWER, 2015; HORÁKOVÁ et al., 2015; LI; ZHOU, 2015; AL-DAGHI et al., 2015; GHANTOUS et al., 2015; PHAM et al., 2015; AMPUERO; ROMERO-GOMES, 2015; GOMES et al., 2006).

O Ministério da Saúde regulamentou a cirurgia bariátrica no Brasil por meio da Portaria $n^{0} 628 / \mathrm{GM}$, de 26 de abril de 2001, considerando como fatores preponderantes que justificam sua homologação: a transformação nos últimos anos dos padrões nutricionais dos brasileiros; a relevância da obesidade na saúde pública; a prevalência da obesidade mórbida; e a verificação de casos que não respondem aos tratamentos convencionais e a necessidade de ampliar a oferta de serviços para realização da gastroplastia (BRASIL, 2001). A cirurgia é indicada quando o indivíduo possui índice de massa corpórea (IMC) igual ou superior a $40 \mathrm{~kg} / \mathrm{m}^{2}$ e tentativa sem sucesso de tratamento clínico por mais de dois anos consecutivos, ou quando se enquadra na classificação de IMC na faixa de 35 a $39,9 \mathrm{~kg} / \mathrm{m}^{2}$, associado a comorbidades (CFM, 2005).

A cirurgia bariátrica é considerada hoje uma ferramenta eficaz no controle e tratamento da obesidade grave, tendo como principais benefícios a perda e manutenção de peso, redução de comorbidades e consequente melhora na qualidade de vida, assim como aceitação da autoimagem e melhor inserção na sociedade. Cerca de $80 \%$ dos pacientes que se submetem à gastroplastia são mulheres em idade fértil (CUENCA, 2014; SHNEIDER, 2014; CASTRO et al., 2013; GRUNDY; WOODCOCK; ETTWOOD, 2008).

No entanto, existe uma lacuna em relação às pesquisas que focam avaliar o perfil nutricional do indivíduo no momento em que busca o serviço para realização do procedimento cirúrgico (LIMA; SAMPAIO, 2007). Sendo assim, o presente estudo teve como objetivo avaliar o perfil socioeconômico e antropométrico de obesas que estão na fila de espera para a realização da cirurgia bariátrica.

\section{METODOLOGIA}

Participaram do presente estudo 100 mulheres obesas que se encontravam na fila de espera para gastroplastia do Centro de Gastroenterologia e Cirurgia da Obesidade - Clínica Bariátrica, que recebe pacientes de Piracicaba (SP) e região, atendidas pelo Sistema Único de Saúde (SUS).

Para inclusão na pesquisa, seguiram-se os seguintes critérios: sexo feminino, idade entre 20 e 45 anos, em menacme, que estão na fila de espera para realização do procedimento de gastroplastia. A escolha do sexo feminino para compor a amostra é explicada 
pela sua maioria nos atendimentos dessa clínica. Este estudo foi submetido e aprovado pelo Comitê de Ética em Pesquisa da Universidade Estadual Paulista "Júlio de Mesquita Filho" (UNESP) Campus de Botucatu (SP), sob o protocolo $\mathrm{n}^{\mathrm{o}} 33 / 2009$, sendo um subprojeto do projeto "Nutrição, obesidade mórbida e cirurgia bariátrica: fatores de suscetibilidade e estudo prospectivo de aspectos genéticos, dietéticos e metabólicos". A faixa etária definida considerou ser uma etapa da vida em que não há processos fisiológicos e hormonais ocorrendo, os quais poderiam não trazer veracidade ao estudo.

O recrutamento da amostra ocorreu via e-mail, de forma aleatória, para pacientes do sexo feminino que estavam no banco de dados da Clínica. Prosseguiu-se através do retorno do contato com agendamento para entrevista.

As participantes que obedeceram aos critérios de inclusão do estudo foram convidadas a participar da pesquisa. Após leitura, explicação e assinatura do termo de consentimento livre e esclarecido, foi feito o preenchimento do questionário da Associação Brasileira de Empresas de Pesquisa (ABEP, 2003) para a divisão das voluntárias em classes econômicas. Esse instrumento considerou o poder de compra por meio dos bens que possuem e o grau de escolaridade do chefe de família. A classificação das mulheres entrevistadas ocorreu por meio de uma pontuação que estimou a renda média familiar e as dividiu em classes (Classe A1: renda média familiar de $\mathrm{R} \$ 7793,00$; Classe A2: renda média familiar de $\mathrm{R} \$ 4.648,00$; Classe B1: renda média familiar de $\mathrm{R} \$$ 2.804,00; Classe B2: renda média familiar de $\mathrm{R} \$ 1.669,00$; Classe C: renda média familiar de $\mathrm{R} \$ 927,00$; Classe D: renda média familiar de $\mathrm{R} \$ 424,00$; Classe E: renda média familiar de $\mathrm{R} \$ 207,00$ ).

Sequencialmente, foram submetidas a uma entrevista para coleta de informações gerais, como endereço, telefone, data de nascimento, idade, estado civil, etnia, profissão, grau de escolaridade, hábito de fumar, consumo de bebida alcoólica, história de peso, tentativas prévias de perda de peso e presença de comorbidades associadas à obesidade.

Para a avaliação antropométrica as variáveis obtidas foram peso e estatura. Os instrumentos utilizados para aferir as medidas foram: balança antropométrica digital (FILIZOLA ${ }^{\circledR}$, São Paulo (SP) - Brasil) com capacidade para $350 \mathrm{~kg}$ devidamente calibrada e estadiômetro fixo
(SECA ${ }^{\circledR}$, Hamburg, Alemanha) constituído por escala métrica. Os procedimentos para aferição dessas medidas seguiram as técnicas propostas por Callaway et al. (1988).

A partir das medidas de peso e estatura foi calculado o índice de massa corpórea (IMC). O valor obtido seguiu os critérios de classificação da Sociedade Americana de Cirurgia Bariátrica (RENQUIST, 2014), sendo considerada obesidade moderada mulheres com IMC 30 a $35 \mathrm{Kg} / \mathrm{m}^{2}$; obesidade grave com IMC 35 a $40 \mathrm{Kg} / \mathrm{m}^{2}$; Obesidade mórbida com IMC 40 a $50 \mathrm{Kg} /$ $\mathrm{m}^{2}$; superobesidade com IMC 50 a $60 \mathrm{Kg} / \mathrm{m}^{2}$ e super/ superobesidade com IMC $>60 \mathrm{Kg} / \mathrm{m}^{2}$.

Os dados obtidos foram tabulados e por meio do programa estatístico BioEstat 5.0, as variáveis foram analisadas de forma descritiva por meio de frequência absoluta e relativa e médias \pm desvio-padrão (DP).

\section{RESULTADOS}

Verificou-se que a idade média das mulheres avaliadas foi de 33,3 $\pm 6,0$ anos. Com relação à faixa etária $28 \%$ tinham de 20 a 29 anos; $55 \%$ de 30 a 39 e $17 \%$ de 40 a 45 anos. Quanto à etnia, a distribuição foi de $50 \%$ branca, $18 \%$ negra e $32 \%$ parda.

Os dados antropométricos apresentaram valores médios \pm DP de peso $119,5 \pm 16,6 \mathrm{Kg}$ e de estatura $1,62 \pm 0,06 \mathrm{~m}$, possibilitando o cálculo do IMC e verificando média \pm DP de $45,75 \pm 6,05 \mathrm{Kg} / \mathrm{m}^{2}$, variando de 35,2 a $72,65 \mathrm{~kg} / \mathrm{m}^{2}$.

A tabela 1 apresenta a classificação das obesas avaliadas segundo o IMC, sendo que houve predomínio da obesidade mórbida segundo a Sociedade Americana de Cirurgia Bariátrica.

Tabela 1. Distribuição do Estado Nutricional das Mulheres Entrevistadas segundo o Índice de Massa Corpórea (IMC)

\begin{tabular}{lcc}
\hline Classificação do IMC* & N & \% \\
\hline Obesidade grave & 13 & 13 \\
Obesidade mórbida & 68 & 68 \\
Superobesidade & 18 & 18 \\
Super/superobesidade & 1 & 1 \\
\hline TOTAL & $\mathbf{1 0 0}$ & $\mathbf{1 0 0}$ \\
\hline
\end{tabular}

*Segundo classificação da Sociedade Americana de Cirurgia Bariátrica. 
A caracterização sociodemográfica das participantes se encontra na tabela 2. Segundo a classificação econômica, a maioria das mulheres pertence à classe C. Quanto ao estado civil e o nível educacional, $45 \%$ são casadas e $66 \%$ das participantes têm escolaridade igual ou superior ao ensino médio completo.

Os trabalhos domésticos (do lar) representam a atividade de maior frequência entre as mulheres (28\%), seguido por diarista (7\%), auxiliar de serviços gerais (6\%), técnica/auxiliar em enfermagem (6), funcionária pública (5\%), costureira (4\%), secretária (4\%), estudante (3\%), dentre outras diversas (37\%).

Tabela 2. Caracterização Sociodemográfica das Participantes da Pesquisa Atendidas pelo Sistema Público de Saúde na Clínica Bariátrica, Piracicaba (SP)

\begin{tabular}{lll}
\hline Variáveis & N & \% \\
\hline Escolaridade & & \\
\hline Fundamental completo/incompleto & 27 & 27 \\
Médio completo/incompleto & 55 & 55 \\
Graduação completa/incompleta & 17 & 17 \\
Pós-Graduação incompleta & 1 & 1 \\
\hline Classificação econômica* & &
\end{tabular}

\begin{tabular}{lcc} 
Classificação econômica* $^{*}$ & & \\
\hline Classe A1 & 1 & 1 \\
Classe B1 & 2 & 2 \\
Classe B2 & 22 & 22 \\
Classe C & 67 & 67 \\
Classe D & 8 & 8 \\
\hline
\end{tabular}

\begin{tabular}{lll}
\hline Estado civil & & \\
\hline Solteira & 27 & 27 \\
União estável & 19 & 19 \\
Casada & 45 & 45 \\
Divorciada & 2 & 2 \\
Separada judicialmente & 6 & 6 \\
Viúva & 1 & 1 \\
\hline
\end{tabular}

* Classe A1: renda média familiar de R\$ 7.793,00; Classe A2: renda média familiar de $\mathrm{R} \$ 4.648,00$; Classe B1: renda média familiar de $\mathrm{R} \$ 2.804,00$; Classe B2: renda média familiar de $\mathrm{R} \$ 1.669,00$; Classe C: renda média familiar de $\mathrm{R} \$ 927,00$; Classe D: renda média familiar de $\mathrm{R} \$ 424,00$; Classe $\mathrm{E}$ : renda média familiar de $\mathrm{R} \$ 207,00$. Classificação Econômica segundo a Associação Brasileira de Empresas de Pesquisas (ABEP).
Quanto aos tratamentos clínicos realizados previamente para tentativa de perda de peso, a associação de dieta, exercício físico e medicamento foram relatados por apenas $\mathbf{5 2} \%$ das mulheres entrevistadas. Em relação ao início da obesidade, $38 \%$ são obesas desde a infância, 9\% relataram o desenvolvimento da obesidade na adolescência, $15 \%$ nas gestações e $38 \%$ na vida adulta.

Quanto ao hábito de fumar e etilismo, 67\% não são tabagistas, $21 \%$ são ex-tabagistas e $12 \%$ são tabagistas atualmente. Apenas 34\% relatam o consumo de bebida alcoólica.

A hipertensão arterial foi a comorbidade de maior predomínio (48\%), seguida por dislipidemias (21\%) e diabetes mellitus (7\%).

\section{DISCUSSÃO}

Há tempos a obesidade não é apenas considerada um problema estético, e sim doença que requer tratamento. A perda de peso pode melhorar as comorbidades, qualidade e expectativa de vida do paciente. No Brasil, há estimativa de que mais de 80 mil pacientes foram submetidos a este tipo de intervenção em 2014, atrás apenas dos Estados Unidos com 140 mil pacientes (RAMOS, 2014; REGINALDO; SILVA, 2014).

Os resultados deste trabalho mostram que 55\% das mulheres que estão à espera pela cirurgia apresentam idade entre 20 a 29 anos e que a média de IMC encontrada $\left(45,75 \pm 6,05 \mathrm{Kg} / \mathrm{m}^{2}\right)$ foi semelhante à encontrada por Lima e Sampaio (2007) $\left(46,5 \pm 7,1 \mathrm{Kg} / \mathrm{m}^{2}\right)$, que avaliaram 50 obesos graves atendidos em três ambulatórios de cirurgia bariátrica da cidade de Fortaleza, dois na esfera pública e um no setor privado. Médias superiores $(52,7 \mathrm{~kg} /$ $\mathrm{m}^{2}$ e $48,51 \mathrm{~kg} / \mathrm{m}^{2}$, respectivamente) foram encontradas nos estudos de Diniz et al. (2008), que avaliaram obesos atendidos pelo sistema público de saúde de Minas Gerais, e no estudo de Petribu et al. (2006) em Recife.

Pelos resultados obtidos neste estudo pôde-se observar que a maioria dos pacientes que procuram o tratamento cirúrgico, o fez antes de apresentarem diagnósticos de superobesidade ou super/ superobesidade. Houve predomínio da obesidade mórbida (68\%) segundo a classificação da Sociedade 
Americana de Cirurgia da Obesidade, uma vez que a maioria das participantes da pesquisa apresentou IMC entre 40 a $50 \mathrm{Kg} / \mathrm{m}^{2}$. Dado semelhante ao encontrado por Lima e Sampaio (2007), onde 70\% da amostra do estudo obtiveram a mesma classificação segundo o IMC. Ramos et al. (2006) apresentaram os resultados iniciais da primeira série de casos brasileiros de cirurgia bariátrica totalmente robótica com 68 pacientes. Destes, 52 eram mulheres com idade média de 40,5 anos e IMC de 41,3 $\mathrm{kg} / \mathrm{m}^{2}$. Em outro estudo brasileiro, $60,6 \%$ dos pacientes à espera do procedimento cirúrgico foram classificados com superobesidade (DINIZ et al., 2008).

Os dados relacionados à profissão mostraram que $28 \%$ das mulheres desenvolvem atividades do lar, enquanto que Porto et al. (2002) relatam $42 \%$ e Kaspary et al. (2006) 37,5\% dessa atividade.

Os resultados deste trabalho mostram que uma mesma proporção de mulheres (38\%) se tornou obesa na infância e na vida adulta. Silva e Nunes (2015) mostraram que mesmo em crianças pobres no Estado do Mato Grosso do Sul, o sobrepeso e a obesidade têm índices alarmantes, somando um total de $23,9 \%$. O excesso de peso em fases precoces da vida pode aumentar o risco de obesidade na fase adulta e para prevenção deveriam ser feitas intervenções focadas em adolescentes e crianças na idade escolar (SHROEDER et al., 2015).

As comorbidades de maior ocorrência observadas neste trabalho foram a hipertensão arterial acometendo 48\% da amostra, seguida de dislipidemias, com $21 \%$ e a presença de diabetes mellitus tipo 2 com $7 \%$. Dados semelhantes foram encontrados em outros estudos no Brasil que apontaram a hipertensão como principal comorbidade em obesos graves (COSTA et al., 2009; DINIZ et al., 2008; ANDERI et al., 2007; RANGEL, et al., 2007). Porto et al. (2002) encontraram que 66\% dos obesos mórbidos do Ambulatório de Obesidade do Hospital Universitário da Universidade Federal da Bahia eram hipertensos, 33,5\% apresentavam dislipidemia e 23,9\% eram diabéticos. Pereira et al. (2015) apontam que a dislipidemia tem uma relação direta com o sobrepeso e obesidade. Segundo os autores, a dislipidemia era 49\% mais prevalente entre pacientes com sobrepeso quando comparados com indivíduos com peso normal, e $83 \%$ mais prevalente entre pacientes obesos. A obesidade é comumente relacionada à presença de inúmeras comorbidades e consequente aumento da mortalidade já que aumenta as chances do paciente desenvolver hipertensão arterial sistêmica, resistência à insulina que é fator de risco de desenvolver diabetes mellitus do tipo 2, alterações nos lipídeos plasmáticos, além de outras comorbidades como apneia do sono, esteatose hepática, alterações respiratórias, infertilidade, infarto e câncer (NASCIMENTO et al., 2015; MANCINI, 2014).

Háinúmeras evidências de quea prática da cirurgia bariátrica pode trazer impactos positivos para o paciente, melhorando a qualidade de vida do obeso mórbido. A obesidade parece estar relacionada com a diminuição da qualidade e da expectativa de vida, podendo reduzir de 5 a 20 anos dependendo do gênero considerado, da raça e da idade. Sendo assim, a cirurgia bariátrica pode ser uma maneira eficaz no tratamento do obeso mórbido levando à redução do peso e das comorbidades associadas. Vale ressaltar ainda a importância de uma equipe multiprofissional na abordagem antes da cirurgia assim como no acompanhamento pós-operatório e esta abordagem deve ser individual e familiar, apontando para o paciente as limitações do tratamento cirúrgico em contraponto de suas expectativas (MANCINI, 2014; CUENCA, 2014; SHNEIDER, 2014).

\section{CONCLUSÃO}

Conclui-se que a maioria das candidatas à cirurgia bariátrica é casada, de etnia branca, têm como principal atividade o cuidado doméstico, têm o segundo grau completo e faz parte da classe $\mathrm{C}$, o que corresponde a uma renda média familiar de $\mathrm{R} \$ 927,00$, segundo critério econômico utilizado no estudo. Na maioria não são etilistas nem tabagistas e atualmente estão com IMC entre 40 e $50 \mathrm{~kg} / \mathrm{m}^{2}$ e já realizaram tentativas prévias de perda de peso. Apesar da maior parte da amostra se encontrar dentro dos padrões para indicação do procedimento cirúrgico, em uma parcela dela, uma busca precoce por essa alternativa ocorre sem que atinjam níveis mais exacerbados de IMC e, em alguns casos, sem a realização de tratamentos clínicos prévios com tratamentos conservadores. 


\section{REFERÊNCIAS}

AL-DAGHRI, N. M.; AL-ATTAS, O. S.; KRISHNASWAMY, S.; MOHAMMED, A. K.; ALENAD, A. M.; CHROUSOS, G. P.; ALOKAIL, M. S. Association of Type 2 Diabetes Mellitus related SNP genotypes with altered serum adipokine levels and metabolic syndrome phenotypes. Int J Clin Exp Med., v. 8, n. 3, p. 4464-71, 2015.

AMPUERO, J.; ROMERO-GOMEZ, M. Prevention of hepatocellular carcinoma by correction of metabolic abnormalities: Role of statins and metformin. World J Hepatol., v. 7, n. 8, p. 1105-11, 2015.

ANDERI JÚNIOR, E.; ARAÚJO, L. G. C.; FUHRO, F. E.; GODINHO, C. A.; HENRIQUES, A. C. Experiência inicial do serviço de cirurgia bariátrica da Faculdade de Medicina do ABC. Arq Med ABC., v. 32, n. 1, p. 25-9, 2007.

ASSOCIAÇÃO BRASILEIRA DE EMPRESAS DE PESQUISA. Critério de Classificação Econômica Brasil. [s.l.; s.n.], 2003.

BRASIL. Ministério da Saúde. Portaria nº 628/GM, de 26 de abril de 2001. Brasília (DF), 2001.

BRASIL. Ministério da Saúde. Secretaria de Vigilância em Saúde. Vigitel Brasil 2013: vigilância de fatores de risco e proteção para doenças crônicas por inquérito telefônico. Ministério da Saúde, Secretaria de Vigilância em Saúde. Brasília: Ministério da Saúde. 2014. 120p.

CALLAWAY, C. W.; CHUMLEA, W. C.; BOUCHARD, C.; HILMES, J. H.; LOHMAN, T. G.; MARTIN, A. D. Circumferences. In: LOHMAN, T. G.; ROCHE, A. F.; MARTORELLI, R. Anthropometric standardization reference manual. Champaign: I.L. Human Kinetics, 1988. p. 39-54.

CASTRO, M. R.; FERREIRA, V. N.; CHINELATO, R. C.; FERREIRA, M. E. Imagem corporal em mulheres submetidas à cirurgia bariátrica: interações socioculturais. Body image in women undergone bariatric surgery: Sociocultural interactions. Motri, v. 9, n. 3, p. 82-95, 2013.

CFM. Conselho Federal de Medicina. Resolução CFM n ${ }^{0}$ $1.766 / 05$ de 11 de julho de 2005. Dispõe sobre normas seguras para o tratamento cirúrgico da obesidade mórbida. Diário Oficial da União, p. 114, seção I, Brasília, 2005.

COSTA, A. C. C.; IVO, M. L.; CANTERO, W. B.; TOGNINI, J. R. F. Obesidade em pacientes candidatos a cirurgia bariátrica. Acta Paul Enferm., v. 22, n. 1, p. 55-9, 2009.

CUENCA, R. M. Qualidade de vida pós-cirurgia bariátrica. Arq Gastroenterol., v. 51, n. 3, p. 163-164, 2014.

DINIZ, M. F. H. S.; PASSOS, V. M. A.; BARRETO, S. M.; DINIZ, M. T. C.; LINARES, D. B.; MENDES, L. N. Perfil de pacientes obesos classe III do sistema público de saúde submetidos à gastroplastia em "Y de Roux", no hospital das clínicas da UFMG: altas prevalências de superobesidade, comorbidades e mortalidade hospitalar. Rev Méd de Minas Gerais, v. 18, n. 3, p. 183-190, 2008.

GHANTOUS, C. M.; AZRAK, Z.; HANACHE, S.; ABOUKHEIR, W.; ZEIDAN, A. Differential role of leptin and adiponectin in cardiovascular system. Int J Endocrinol., 2015. doi: 10.1155/2015/534320, 2015.

GOMES, M. B.; NETO, D. G.; MENDONÇA, E.; TAMBASCIA, M. A.; FONSECA, R. M.; RÉA, R. R. Prevalência de sobrepeso e obesidade em pacientes com diabetes mellitus do tipo 2 no Brasil: estudo multicêntrico nacional. Arq Bras Endocrinol Metab., v. 50, n. 1, p. 136-144, 2006.

GRUNDY, M. A.; WOODCOCK, S.; ETTWOOD, S. E. The surgical management of obesity in young woman: Consideration of the mother's and baby's health before, during, and after pregnancy. Surg Endosc., v. 22, n. 10, p. 2107-2226, 2008. doi: 10.1007/s00464-008-0019-5

HORÁKOVÁ,D.;AZEEM, K.; BENEŠOVÁ, R.; PASTUCHA, D.; HORÁK, V.; DUMBROVSKÁ, L.; MARTÍNEK, A.; NOVOTNÝ, D.; ŠVAGERA, Z.; HOBZOVÁ, M.; GALUSZKOVÁ, D.; JANOUT, V.; DONĚVSKÁ, S.; VRBKOVÁ, J.; KOLLÁROVÁ, H. Total and High Molecular Weight Adiponectin Levels and Prediction of Cardiovascular Risk in Diabetic Patients. Int J Endocrinol., 2015. doi: 10.1155/2015/545068.

KASPARY, N.; SOARES, A.; GUIMARÃES, A. C. A.; SIMAS, J. P. N. Estilo de vida de mulheres adultas obesas da cidade de Cruz Alta-RS. Rev Ed Física, v. 17, n. 2, p. 193-201, 
2006.

LI, L.; HARDY, R.; KUH, D.; POWER, C. Life-course body mass index trajectories and blood pressure in midlife in two British birth cohorts: stronger associations in the later-born generation. Int J Epidemiol., v. 44, n. 3, 2015.

LI, Y. X.; ZHOU, L. Vitamin D Deficiency, Obesity and Diabetes. Cell Mol Biol., v. 61, n. 3, p. 35-8, 2015.

LIMA, L. P.; SAMPAIO, H. A. C. Caracterização socioeconômica, antropométrica e alimentar de obesos graves. Cienc Saúde Colet., v. 12, n. 4, p. 1011-1020, 2007.

MANCINI, M. C. Bariatric surgery - An update for the endocrinologist / Cirurgia bariátrica - Uma atualização para o endocrinologista. Arq Bras Endocrinol Metab., v. 58, n. 9 , p. 875-888, 2014.

MARQUES, R. C. A.; GABBIATTI, G. C.; GRAVENA, A. A. F.; AMARAL, V. A. Influência das dietas hipercalóricas sobre os parâmetros de obesidade, dislipidemia e hiperglicemia em ratos. Saúde e Pesquisa, v. 8, n. 1, p. 55-62, jan./ abr. 2015. DOI: <http://dx.doi.org/10.17765/19831870.2015v8n1p55-62>.

NASCIMENTO, F. V.; PICCOLI, V.; BEER, M. A.; VON FRANKENBERG, A. D.; CRISPIM, D.; GERCHMAN, F. Association of HSD11B1 polymorphic variants and adipose tissue gene expression with metabolic syndrome, obesity and type 2 diabetes mellitus: a systematic review. Diabetol Metab Syndr., v. 7, n. 38, 2014.

NOVAIS, P. F, S.; RASERA JUNIOR, I.; LEITE, C. V. S.; OLIVEIRA, M. R. M. Evolução e classificação do peso corporal em relação aos resultados da cirurgia bariátrica: derivação gástrica em Y de Roux. Arq Bras Endocrinol Metab., v. 54, n. 3, p. 303-310, 2010.

PEREIRA, L. P.; SICHIERI, R.; SEGRI, N. J.; SILVA, R. M.; FERREIRA, M. G. Self-reported dyslipidemia in central-west Brazil: prevalence and associated factors. Cien Saude Colet., v. 20, n. 6, p. 1815-1824, 2015.

PETRIBU, K.; RIBEIRO, E. S.; OLIVEIRA, F. M. F.; BRAZ, C.
I. A.; GOMES, M. L. M.; ARAUJO, D. E.; ALMEIDA, N. C. N.; ALBUQUERQUE, P. C.; FERREIRA, M. N. L. Transtornos de Compulsão Alimentar Periódica em uma População de Obesos Mórbidos Candidatos a Cirurgia Bariátrica do Hospital Universitário Oswaldo Cruz, em Recife - PE. Arq Bras Endocrinol Metab., v. 50, n. 5, p. 775-782, 2006.

PHAM, I.; COSSON, E.; NGUYEN, M. T.; BANU, I.; GENEVOIS, I.; POIGNARD, P.; VALENSI, P. Evidence for a Specific Diabetic Cardiomyopathy: An Observational Retrospective Echocardiographic Study in 656 Asymptomatic Type 2 Diabetic Patients. Int J Endocrinol., DOI: $10.1155 / 2015 / 743503$.

PORTO, M. C. V.; BRITO, A. D. F.; AMORAS, M.; VILLELA, N. B.; ARAÚJO, L. M. B. Perfil do Obeso Classe III do Ambulatório de Obesidade de Um Hospital Universitário de Salvador, Bahia. Arq Bras Endocrinol Metab., v. 46, n. 12, p. 668-673, 2002.

RAMOS, A. C. Brazil looking for completing his space in bariatric surgery / O Brasil procurando preencher o seu espaço na cirurgia bariátrica. ABCD, Arq Bras Cir Dig., v. 27, n. 1, p. 01-01, 2014.

RAMOS, A. C.; DOMENE, C. E.; VOLPE, P.; PAJECKI, D.; D’ALMEIDA, L. A. V.; RAMOS, M. G.; BASTOS, E. L. S.; KIM, K. C. Resultados iniciais da primeira série de casos brasileira de cirurgia bariátrica totalmente robótica / Early outcomes of the first Brazilian experience in totally robotic bariatric surgery. ABCD, Arq Bras Cir Dig., v. 26, supl. 1, p. 2-7, 2006.

RANGEL, L. O. B.; FARIA, V. S. P.; MAGALHÃES, E. A.; ARAÚJO, A. C. T.; BASTOS, E. M. R. D. Perfil de saúde e nutricional de pacientes portadores de obesidade mórbida candidatos à cirurgia bariátrica. Rev Bras Nutr Clin., v. 22, n. 3, p. 214-9, 2007.

REGINALDO, G. J.; SILVA, A. F. Carência de vitamina B12 após cirurgia bariátrica no método BGYR. Saúde e Pesquisa, v. 7, n. 3, p. 487-494, set./dez. 2014.

RENQUIST, K. Obesidade classification. Obes Surg., v. 8, n. 4, p. 480, 1998.

RIGBY, N. Eating and obesity-the new world disorder. 
Nutrients, v. 5, n. 10, p. 4206-10, 2013.

RUIZ, E.; ÁVILA, J. M.; VALERO, T.; DEL POZO, S.; RODRIGUEZ, P.; ARANCETA-BARTRINA, J.; GIL, Á.; GONZÁlEZ-GROSS, M.; ORTEGA, R. M.; SERRAMAJEM, L.; VARELA-MOREIRAS, G. Energy Intake, Profile, and Dietary Sources in the Spanish Population: Findings of the ANIBES Study. Nutrients., v. 7, n. 6, p. 4739-62, 2015.

SCHNEIDER, D. S. Qualidade de vida de pacientes antes e depois de cirurgia bariátrica. Acta Paul Enferm., v. 27, n. 2, p. 01-02, 2014.

SCHROEDER, N.; RUSHOVICH, B.; BARTLETT, E.; SHARMA, S.; GITTELSOHN, J.; CABALLERO, B. Early Obesity Prevention: A Randomized Trial of a PracticeBased Intervention in 0-24-Month Infants. J Obes., 2015. DOI:10.1155/2015/795859.

SILVA, D. A.; NUNES, H. E. Prevalence of underweight, overweight and obesity in poor children from Mato Grosso do Sul. Rev Bras Epidemiol., v. 18, n. 2, p. 46675, 2015.

VARELA-MOREIRAS, G.; ALGUACIL MERINO, L. F.; APERTE, E. A.; BARTRINA, J. A.; AVILA TORRES, J. M.; LAÍN, A. S.; CORTÉS, S. B.; GARCÍA, L. C.; DAL RE SAAVEDRA, M. A.; RUBIO, A. R. Obesity and sedentarism in the 21st century: what can be done and what must be done? Nutr Hosp., v. 28, n. 1, p. 1-12, 2013.

WORLD HEALTH ORGANIZATION. Obesity and overweight: what are overweight and obesity? 2006. Disponível em: <http://www.who.int/mediacentre> Acesso em: 05 jun. 2015.

Recebido em: 07 de julbo de 2015 Aceito em: 14 de setembro de 2015 